\title{
SPECTRAL THEORY AND REPRESENTATIONS OF NILPOTENT GROUPS
}

\author{
P. LEVY-BRUHL, A. MOHAMED, AND J. NOURRIGAT
}

\begin{abstract}
We give an estimate of the number $N(\lambda)$ of eigenvalues $<\lambda$ for the image under an irreducible representation of the "sublaplacian" on a stratified nilpotent Lie algebra. We also give an estimate for the trace of the heat-kernel associated with this operator. The estimates are formulated in term of geometrical objects related to the representation under consideration. An important particular case is the Schrödinger equation with polynomial electrical and magnetical fields.
\end{abstract}

\section{INTRODUCTION}

We first consider the Schrödinger operator with polynomial electrical and magnetical fields

$$
P=\sum_{j=1}^{n}\left(D_{j}-A_{j}(x)\right)^{2}+V(x),
$$

where $A_{j}$ and $V$ are real polynomials on $\mathbb{R}^{n}$, of degree $\leq r$, and with $V \geq 0$. We define $B_{j k}=\partial A_{j} / \partial x_{k}-\partial A_{k} / \partial x_{j}$ and assume that there is no rotation of the coordinates axis making all the $B_{j k}$ and $V$ independent of one of the $x_{i}$ 's. This case has been investigated in [9]: define

$$
\begin{gathered}
M(x)=\sum_{|\alpha| \leq r}\left|\partial^{\alpha} V(x)\right|^{1 /(|\alpha|+2)}+\sum_{\alpha, j, k}\left|\partial^{\alpha} B_{j k}(x)\right|^{1 /(|\alpha|+2)}, \\
M(x, \xi)=|\xi|+M(x) .
\end{gathered}
$$

Let $N(\lambda)$ be the number of eigenvalues of $P<\lambda$ and $N_{0}(\lambda)$ the volume in $\mathbb{R}^{2 n}$ of the set of points $(x, \xi)$ such that $M(x, \xi)^{2} \leq \lambda$. Then there is a $C>1$, independent of $\lambda$ such that $C^{-1} N_{0}\left(C^{-1} \lambda\right) \leq N(\lambda) \leq C N_{0}(C \lambda)$ for all $\lambda>0$. A more precise equivalent in particular cases is given in [13]. This result was proved before, in a different formulation, by Fefferman, when the $A_{j}$ 's are zero [1]. If $V$ is the square of a polynomial, $P$ is of the form $\pi(-\Delta)$ where $\Delta$ is a sublaplacian on a stratified $r$-step nilpotent Lie group and $\pi$ an irreducible representation $[2,9]$. In the general case, $(V \geq 0)$, our following results needs

Received by the editors May 15, 1991 and, in revised form, September 24, 1991.

1991 Mathematics Subject Classification. Primary 35P20, 22 E27.

Key words and phrases. Representations of nilpotent Lie groups, spectral theory for partial differential equations.

The results have been presented at the 1991 meeting on "Harmonic analysis and representation theory" (R. S. Howe. org.) (held January 27 to February 2), in Oberwolfach, Germany. 
a very small change to be applied. This example is one of the motivations for the present generalization.

\section{Statement of the Results}

Let $\mathfrak{G}$ be a stratified $r$-steps nilpotent Lie algebra. In other words, we assume that $\mathfrak{G}$ can be written as a direct sum of subspaces $\mathfrak{G}_{j} \quad(1 \leq j \leq r)$ such that

$$
\begin{gathered}
{\left[\mathfrak{G}_{j}, \mathfrak{G}_{k}\right] \subset \mathfrak{G}_{j+k} \quad \text { if } j+k \leq r,} \\
{\left[\mathfrak{G}, \mathfrak{G}_{k}\right]=0 \quad \text { if } j+k>r,}
\end{gathered}
$$

and such that $\mathfrak{G}$ is generated as Lie algebra by the subspace $\mathfrak{G}_{1}$. We choose a basis $X_{1}, \ldots X_{p}$ of $\mathfrak{G}_{1}$, and we shall be interested in spectral properties of the image under irreducible representations of the sublaplacian $\Delta=-\sum_{j=1}^{p} X_{j}^{2}$.

Let $\pi$ be an irreducible, unitary, nontrivial representation of the connected simply connected group $\exp \mathfrak{G}$ associated to $\mathfrak{G}$. It is well known that the operator $\pi(-\Delta)$, defined on the space $\mathscr{S}_{\pi}$ of $C^{\infty}$ vectors of the representation $\pi$, has a unique selfadjoint extension, still denoted $\pi(-\Delta)$, whose spectrum is a sequence $\left(\lambda_{j}\right) \quad(j \in \mathbb{N})$ of positive eigenvalues, such that $\lambda_{j} \leq \lambda_{j+1}$ and $\lambda_{j} \rightarrow+\infty$. Let us denote by $N(\lambda)$ the number of eigenvalues $\lambda_{j} \leq \lambda$. Our first goal is to give an estimate for $N(\lambda)$ in terms of geometrical objects associated to the representation $\pi$.

We denote by $\delta_{t}(t>0)$ the natural dilations of $\mathfrak{G}$; that is, the linear maps defined by $\delta_{t}(X)=t^{j} X$ if $X \in \mathfrak{G}_{j}$. Let also $\mathfrak{G}^{*}$ be the dual of $\mathfrak{G}, \delta_{t}^{*}$ the transpose of $\delta_{t}$, and \|\|$\|$ a homogeneous norm on $\mathfrak{G}^{*}$, i.e., a nonnegative continuous, subadditive function on $\mathfrak{G}^{*}$, only vanishing at the origin and satisfying $\left\|\delta_{t}^{*} l\right\|=t\|l\|$ if $t>0$ and $l \in \mathfrak{G}^{*}$.

By the Kirillov theory, the representation $\pi$ is associated with an orbit $O_{\pi}$ of the coadjoint representation of the group $G=\exp \mathfrak{G}$ in $\mathfrak{G}^{*}$. The $G$ invariant measures on $O_{\pi}$ are proportional, and we denote by $\mu$ the canonical one; that is, with the correct normalization for the character formula [4, 12]. For each $\lambda>0$, we set

$$
N_{0}(\lambda)=\mu\left(l \in O_{\pi},\|l\|^{2} \leq \lambda\right) .
$$

We can now state the estimate for $N(\lambda)$.

Theorem 1. There exists a constant $C>1$, independent of $\lambda$, and of the representation $\pi$ such that

$$
C^{-1} N_{0}\left(C^{-1} \lambda\right) \leq N(\lambda) \leq C N_{0}(C \lambda), \quad \text { for all } \lambda>0 .
$$

Manchon gives in [7] the proof of a conjecture of Karasev-Maslov [3]. His result does not apply to our operator, but rather to $-\sum_{j} Y_{j}^{2}$, where $\left(Y_{j}\right)$ is a basis of $\mathfrak{G}$ as a vector space. Such an operator is also selfadjoint with compact resolvent, and $N(\lambda)$ is given by a Weyl type formula. Such a formula does not make sense in general in our case, the integral involved in it being nonconvergent.

It is also well known, since $\pi$ is irreducible, that for $t>0$, the operator $\exp (-t \pi(\Delta))$ is trace-class. We can estimate its trace

$$
Z(t)=\operatorname{Tr}(\exp (-t \pi(\Delta)))
$$

by the following result, using the function

$$
Z_{0}(t)=\int_{\mathscr{O}_{\pi}} \exp \left(-t\|l\|^{2}\right) d \mu(l) .
$$


Theorem 2. There exists a constant $C>1$, independent of $t>0$, and of the representation $\pi$ such that

$$
C^{-1} Z_{0}(C t) \leq Z(t) \leq C Z_{0}\left(C^{-1} t\right) .
$$

As noticed in the introduction, our results can be used for the Schrödinger operator with polynomial electrical and magnetical fields.

\section{SKETCH OF THE PROOF}

Remark. We give here a sketch of the proof of Theorem 1. The reader may consult [6] for the details.

We construct Hilbert spaces of sequences controlling the norm of the Sobolev spaces associated with $\pi(-\Delta)$ and use the minimax formula.

For this construction, we need a suitable set of functions in $L^{2}\left(\mathbb{R}^{n}\right)$. The properties of these functions have been suggested both by the ideas of Perelomov [11] (coherent states) and of Meyer [8] (wavelets).

We realize the representation $\pi$ in the following form: for $X \in \mathfrak{G}, \pi(X)$ is a differential operator on $\mathbb{R}^{n}$,

$$
\begin{aligned}
\pi(X)= & A_{1}(X) \partial / \partial x_{1}+A_{2}\left(x_{1}, X\right) \partial / \partial x_{2} \\
& +\cdots+A_{n}\left(x_{1}, \ldots, x_{n-1}, X\right) \partial / \partial x_{n}+i B(x, X),
\end{aligned}
$$

where the $A_{j}$ and $B$ are real linear forms in $X$ and polynomials in $x \in \mathbb{R}^{n}$, $A_{1}$ is independent of $x$, and $A_{j}$ only depends on $\left(x_{1}, \ldots, x_{j-1}\right)$. For every finite sequence $I=\left(i_{1}, \ldots, i_{m}\right)$ of positive integers smaller than $p, \pi\left(X_{I}\right)$ is the iterated commutator

$$
\pi\left(X_{I}\right)=\left(\operatorname{ad} \pi\left(X_{i_{1}}\right)\right) \cdots\left(\operatorname{ad} \pi\left(X_{i_{m-1}}\right)\right) \pi\left(X_{i_{m}}\right) .
$$

Let $\pi(X)(x, \xi)$ be the complete symbol of the operator $\pi(X)$. The orbit $\mathscr{O}_{\pi}$ is the set of linear forms $X \rightarrow-i \pi(X)(x, \xi)$ for $(x, \xi)$ in $\mathbb{R}^{2 n}$, and the canonical measure $\mu$ is then $d x d \xi$. We can also choose the homogeneous norm

$$
\|l\|=\sum\left|l\left(X_{i}\right)\right|^{1 /|I|} .
$$

The functions $M_{\pi}(x, \xi)$ and $M_{\pi}(x)$ are defined by

$$
M_{\pi}(x, \xi)=\sum_{|I| \leq r}\left|\pi\left(X_{I}\right)(x, \xi)\right|^{1 /|I|} \text { and } M_{\pi}(x)=\inf _{\xi} M_{\pi}(x, \xi) .
$$

For the representation $\pi$ under consideration, $M_{\pi}(x)>0$. We prove the theorems using this "concrete" realization of $\pi$. We are not able to construct an orthonormal basis of $L^{2}$ so that the Sobolev spaces associated to our operator are $l^{2}$ weighted spaces, but the following result is a substitute and allows the use of minimax formulas. Let $p(x, \xi)=x,(x, \xi) \in \mathbb{R}^{n}$. For every finite sequence $\left(\alpha_{1}, \ldots, \alpha_{m}\right)$ of positive integers smaller than $p$, we define $\pi\left(X^{\alpha}\right)=$ $\pi\left(X_{\alpha_{1}}\right) \cdots \pi\left(X_{\alpha_{m}}\right)$, and for

$$
u \in C_{0}^{\infty}\left(\mathbb{R}^{n}\right):\|u\|_{m, \pi}=\left(\sum_{|\alpha|=m}\left\|\pi\left(X^{\alpha}\right) u\right\|^{2}\right)^{1 / 2} .
$$

Theorem 3. "Coherent states." Let $\pi$ be an induced representation of $\mathfrak{G}$ realized in the form $(*)$ with $M_{\pi}(x)>0$ for all $x \in \mathbb{R}^{n} . \quad(\pi$ is not necessarily 
irreducible.) For every $a>0$ small enough, there exists $\left(\psi_{j}\right)$ and $\left(\Psi_{j}\right) \quad(j \in \mathbb{N})$ in $C_{0}^{\infty}\left(\mathbb{R}^{n}\right)$, a sequence $\Omega_{j}$ of compact sets in $\mathbb{R}^{2 n}$, a point $\left(x_{j}, \xi_{j}\right) \in \Omega_{j}$, and $C>0$ with:

1. $u=\sum_{j}\left(u, \Psi_{j}\right) \psi_{j}$ for all $u$ in $L^{2}\left(\mathbb{R}^{n}\right)$.

2. $\left(\operatorname{supp} \Psi_{j}\right) \subset(\operatorname{supp}) \psi_{j} \subset p\left(\Omega_{j}\right)$ for all $j \in \mathbb{N}$.

3. Every finite subset $A$ of $\mathbb{N}$ contains a part $B$ such that $\# B \geq(\# A) / C$ and such that for every sequence $\left(\lambda_{j}\right)$ of complex numbers

$$
\sum_{j \in B}\left|\lambda_{j}\right|^{2} \leq C\left\|\sum_{j \in B} \lambda_{j} \psi_{j}\right\|^{2} .
$$

4. For every integer $m \geq 0$, there exists $C_{m}>0$ with the properties: If $u \in C_{0}^{\infty}\left(\mathbb{R}^{n}\right)$ is of the form $u=\sum_{j} \lambda_{j} \psi_{j} \quad\left(\lambda_{j} \in \mathbb{C}\right.$ not necessarily given by $\left.\left(u, \Psi_{j}\right)\right)$ then $\|u\|_{m, \pi}^{2} \leq C_{m} \sum_{j}\left|\lambda_{j}\right|^{2} M_{\pi}\left(x_{j}, \xi_{j}\right)^{2 m}$. If in addition $\lambda_{j}=\left(u, \Psi_{j}\right)$ then we have $\sum_{j}\left|\left(u, \Psi_{j}\right)\right|^{2} M_{\pi}\left(x_{j}, \xi_{j}\right)^{2 m} \leq C_{m}\|u\|_{m, \pi}^{2}$.

5. $\mathbb{R}^{2 n} \subset \bigcup_{j}\left(\Omega_{j}\right)$ and every $(x, \xi)$ is contained in at most $C$ of the $\Omega_{j}$ 's. Further, $1 / C \leq \operatorname{Vol}\left(\Omega_{j}\right) \leq C$.

6. For $(x, \bar{\xi})$ in $\Omega_{j}$, we have the estimate $(1 / C) M_{\pi}\left(x_{j}, \xi_{j}\right) \leq M_{\pi}(x, \xi) \leq$ $C M_{\pi}\left(x_{j}, \xi_{j}\right)$.

7. $C$ and the constants $C_{m}$ only depend on $a$ and $\mathfrak{G}$ not on $\pi$.

The construction of the coherent states is first performed locally: we associate to each $x \in \mathbb{R}^{n}$ a representation $\sigma_{x}$, equivalent to $\pi$ such that $\sigma_{x}$ has the form (*) with $M_{\sigma_{x}}(0)=M_{\pi}(x)$ and the coefficients of the polynomials in the expression of $\sigma_{x}\left(\delta_{t}^{-1}(X)\right)$ where $t=M_{\pi}(x)$ are bounded, independently of $x$ and $\pi$. Using Fourier series and induction on the dimension $n$, "local coherent states" for $\sigma_{x}$ are constructed. Certain properties in Theorem 3 are only satisfied if $u$ is supported in the ball of radius $a$, centered at 0 . The coherent states are then obtained by means of a partition of unity, modeled on the symplectic diffeomorphisms related to the intertwining operators between $\pi$ and $\sigma_{x}$.

In the proof, the following theorem is used (the notation is as before).

Proposition 4. There exists $C>0$ such that for every representation $\pi$ of the form $(*)$ and all $u \in S\left(\mathbb{R}^{n}\right)$

$$
\sum_{|\alpha| \leq m}\left\|M_{\pi}(x)^{m-|\alpha|} \pi\left(X^{\alpha}\right) u\right\|^{2} \leq C\|u\|_{m, \pi}^{2} .
$$

In this form, this theorem requires $\mathfrak{G}$ to be stratified, however, the proof of Lemma 4.5 in [10] works without this hypothesis. The function $M$ is then more complicated, reflecting the structure of $\mathfrak{G}$. It is possible to formulate our present results in this set-up. The same method also applies to more general operators than the model presented here; we need only a control of $(\pi(P) u, u)$ in terms of the \|\|$_{m, \pi}$ norm.

\section{REFERENCES}

1. C. L. Fefferman, The uncertainty principle, Bull. Amer. Math. Soc. 9 (1983), 129-206.

2. B. Helffer and J. Nourrigat, Hypoellipticité maximale pour des opérateurs polynômes de champs de vecteurs, Progr. Math., vol. 58, Birkhauser, Boston, MA, 1985. 
3. Karasev and V. Maslov, Algebras with general commutation relations and their applications II, J. Soviet Math. 15 (3) (1981), 273-368.

4. A. Kirillov, Unitary representations of nilpotent groups, Russian Math. Survey 14 (1962), 53-104.

5. P. G. Lemarié, Base d'ondelettes sur les groupes de Lie stratifiés, Bull. Soc. Math. France 117 (1989), 211-232.

6. P. Lévy-Bruhl, A. Mohamed and J. Nourrigat, Etude spectale d'opérateurs sur des groupes nilpotents, Séminaire "Equations aux Dérivées Partielles”, École Polytechnique (Palaiseau), Exposé 18, 1989-90; preprint, 1991.

7. D. Manchon, Formule de Weyl pour les groupes de Lie nilpotents, Thèse, Paris, 1989.

8. Y. Meyer, Ondelettes et opérateurs, Hermann, Paris, 1990.

9. A. Mohamed and J. Nourrigat, Encadrement du $N(\lambda)$ pour des opérateurs de Schrödinger avec champ magnétique, J. Math. Pures Appl. (9) 70 (1991), 87-99.

10. J. Nourrigat, Inégalités $L^{2}$ et représentations de groupes nilpotents, J. Funct. Anal. 74 (1987), 300-327.

11. A. Perelomov, Generalized coherent states and their applications, Texts Monographs Phys., Springer, 1981.

12. L. Pukanszki, Leçons sur les représentations des groupes, Dunod, Paris, 1967.

13. B. Simon, Non classical eigenvalue asymptotics, J. Funct. Anal. 53 (1983), 84-98.

(P. Levy-Bruhl and J. Nourrigat) Departement de Mathematiques, Universite de Reims, B. P. 347,51062 Reims CEDEX, France

(A. Mohamed) Department de Mathematiques, Universite de Nantes, 2 rue de la Houssinière, 44072 Nantes CEDEX, France 